\title{
The OLETF Rat as a Model of Central Sensitization: Possible Relevance to the Study of Fibromyalgia and Related Diseases
}

\author{
Francisco Hélder Cavalcante Félix ${ }^{1, *}$ and Juvenia Bezerra Fontenele ${ }^{2}$ \\ ${ }^{I}$ Department of Biophysiology and Pharmacology, Faculdade de Medicina de Juazeiro do Norte, \\ Av. Leão Sampaio Km 3 S/N, 63040 000, Juazeiro do Norte, CE, Brazil \\ ${ }^{2}$ Faculdade Católica Rainha do Sertão, Juvêncio Alves, 660, 63900000, Quixadá, CE, Brazil
}

Received August 31, 2007

Keywords: fibromyalgia, Otsuka Long-Evans Tokushima Fatty (OLETF) rat, nociception test

Fibromyalgia syndrome (FMS) is a musculoskeletal diffuse pain condition with associated hyperalgesia and allodynia, responsible for a great social and economical burden. Its physiopathogenesis is uncertain. The main theory explaining it (central sensitization model, CSM) relates to many chronic pain states.

The CSM actually labels not a single unified hypothesis but, rather, a group of evidence-based central nervous system (CNS)-centered physiopathogenesis hypotheses for FMS. It constitutes the only model capable of explaining the majority of symptoms of FMS and predicts that CNS-acting agents are the most effective drugs for its treatment. In spite of its success, many questions remain to be clarified. One of the drawbacks of FMS research is the fact that there is no experimental model of central sensitization (CS).

Miyasaka et al. (1) reported nociception and neural pain processing abnormalities in the Otsuka Long-Evans Tokushima Fatty (OLETF) rat, a spontaneously cholecystokinin A receptor (CCK-AR) knockout rat strain that has many features of metabolic syndrome and is used as a type II diabetes (DMII) model. OLETF rats showed shortening of withdrawal latency to thermal pain in the plantar test compared to control rats, indicating thermal hyperalgesia. Behavioral responses following paw withdrawal were disturbed in OLETF rats. The 5hydroxytryptamine (5-HT) and 5-hydroxyindole acetic acid contents in the hippocampus and frontal cortex of OLETF rats were significantly higher than in those of the controls.

Interest has been growing in a possible involvement of 5-HT in FMS. Strong evidence has accumulated to support the hypothesis that an alteration in 5-HT neuronal functioning might be related to the pathophysiology of FMS. Serotonergic neurotransmission has

*Corresponding author. helderfelix@uol.com.br

Published online in J-STAGE

doi: 10.1254/jphs.LT0070011 a significant function in nociception; alterations in 5-HT metabolism and transmission might therefore be important in the pathogenesis of FM. Aberrant pain perception in FM may result from instability of the 5-HT system in FM. On this basis, drugs acting on 5-HT receptors, in particular on $5-\mathrm{HT}_{2}$ and $5-\mathrm{HT}_{3}$, are being used or investigated for the clinical management of FMS (2). Thus, the alterations in nociception (hyperalgesia) and in painassociated behavior in OLETF rats, coupled to the brain 5-HT alterations observed in these animals, make them good candidates for the first CS and FMS animal model.

Recent publications suggest that FMS patients have more frequently metabolic syndrome, and a hyperadrenergic state with blunted epinephrine secretion (3). In addition, elevated body mass index (BMI) correlated with secondary FMS (4). These and other recent observations would suggest that FMS and associated CS could have some relation to cardiovascular alterations and to cardiovascular diseases and co-morbidities. The fact that a DMII animal model shows nociception alterations that resemble those of the CS of FMS may be additional evidence for some correlation.

\section{References}

1 Miyasaka K, Nomoto S, Ohta M, Kanai S, Kaneko T, Tahara S, et al. Disturbance to response to acute thermal pain in naturraly occurring cholecystokinin-A receptor gene knockout Otsuka Long-Evans Tokushima Fatty (OLETF) rats. J Pharmacol Sci. 2006;101:280-285.

2 Bazzichi L, Giannaccini G, Betti L, Mascia G, Fabbrini L, Italiani $\mathrm{P}$, et al. Alteration of serotonin transporter density and activity, in fibromyalgia. Arthritis Res Ther. 2006;8:R99.

3 Loevinger BL, Muller D, Alonso C, Coe CL. Metabolic syndrome in women with chronic pain. Metabol Clin Exp. 2007;56:87-93.

4 Chaiamnuay S, Bertoli AM, Fernández M, Apte M, Vilá LM, Reveille JD, et al. The impact of increased body mass index on systemic lupus erythematosus. Data from LUMINA, a multiethnic cohort. J Clin Rheumatol. 2007;13:128-133. 